\title{
Editorial: Microbial Landscape Ecology: Highlights on the Invisible Corridors
}

\author{
Cendrine Mony ${ }^{1 *}$, Brendan J. M. Bohannan ${ }^{2}$, Mathew A. Leibold ${ }^{3}$, Kabir Peay ${ }^{4}$ and \\ Philippe Vandenkoornhuyse ${ }^{1}$ \\ 1 Université de Rennes 1, CNRS, UMR6553 ECOBIO, Rennes, France, ' Center for Ecology and Evolutionary Biology, \\ University of Oregon, Eugene, OR, United States, ${ }^{3}$ Department of Biology, University of Florida, Gainesville, FL, \\ United States, ${ }^{4}$ Department of Biology, Stanford University, Stanford, CA, United States
}

Keywords: landscape ecology, spatial ecology, metacommunity, assembly rule, microorganisms

\section{Editorial on the Research Topic}

Microbial Landscape Ecology: Highlights on the Invisible Corridors

\section{INTRODUCTION}

Understanding community assembly is a key question in ecology (Kraft and Ackerly, 2014). The first integration of spatial processes at large spatial scales was introduced via the island biogeography theory of MacArthur and Wilson (1967), while landscape ecology emerged in the early 1990's as a new way for analyzing the causes and consequences of spatial patterns across landscapes (Wiens et al., 1993; Turner et al., 2001). In parallel, the consideration of species dynamics as resulting from the interrelationships among local populations, and individual fluxes among them

OPEN ACCESS

Edited and reviewed by: Peter Convey, British Antarctic Survey (BAS), United Kingdom

${ }^{*}$ Correspondence: Cendrine Mony cendrine.mony@univ-rennes1.fr

Specialty section:

This article was submitted to Biogeography and Macroecology, a section of the journal Frontiers in Ecology and Evolution

Received: 04 August 2021 Accepted: 30 August 2021 Published: 01 October 2021

Citation:

Mony C, Bohannan BJM, Leibold MA, Peay $K$ and Vandenkoornhuyse $P$ (2021) Editorial: Microbial Landscape Ecology: Highlights on the Invisible Corridors. Front. Ecol. Evol. 9:753213. doi: 10.3389/fevo.2021.753213 led to the metapopulation concept (Hanski, 1994), extended 20 years ago to the metacommunity concept (Leibold et al., 2004). This framework based on a more mechanistic understanding of species distribution does not integrate space in an explicit manner, but considers dispersal fluxes as a proxy for population isolation. These two main conceptual streams have converged recently to allow better prediction of community assembly at the landscape level.

The application of these concepts to microbes has been slow to develop, due to our limited understanding of microbial habitat requirements, the species concept used, and our limited capacity for spatially extensive surveys of microbial distributions. In addition, there is a long-held assumption that microbial taxa have no meaningful dispersal limits because of their small size and high propagule production (Baas-Becking's hypothesis; Baas Becking, 1934). If unlimited passive dispersion of microorganisms is occurring, biotic and abiotic environmental filters would explain the observed high heterogeneity in the microbial communities in many (all) ecosystems. These views have been challenged by more recent evidence demonstrating strong dispersal limitation, and biogeography has consequently become more prominently used for describing and understanding large-scale spatial patterns (Martiny et al., 2006; Hanson et al., 2012; Donaldson et al., 2016).

Understanding the landscape-level drivers of microbiota complexity is important in a broader ecological context because of the tremendous role these microorganisms play in many ecological functions (e.g., carbon and nutrient cycles, resistance of organisms to stresses, behaviors, and reproduction, etc.). Despite the limitation of mass sequencing approaches in species detection, their application has resulted in the observation that microbial communities encompass tremendous taxonomic diversity, including bacteria, archaea, fungi, and protozoa. The microbial world is also characterized by a large range of life-styles, from free-living organisms to microorganisms associated with plant, animal, and human hosts. Landscape drivers may shape community assembly 
either directly through metapopulation and metacommunity processes or indirectly through host-distribution, although these relationships and their related mechanisms are still poorly characterized, and have mostly focused on pathogens.

Overall the present topic addressed how landscape ecology including metapopulation and metacommunity frameworks can apply successfully to microorganisms. Through a large range of microorganism types and ecosystems considered, we provide a set of papers in this emerging field of microbial landscape ecology. These are categorized in three main topics: biogeography, landscape ecology, and metacommunity ecology. A review of this existing state of the art and introduction to main concepts are presented in this topic in Mony et al.

\section{BIOGEOGRAPHY}

Microorganisms are highly heterogeneous in space from large to small spatial scales (e.g., Bahram et al., 2015). Biogeography analyzes the spatial patterns at large scale and aims at determining the environmental factors shaping these spatial patterns. The development of biogeography for microorganisms is recent (e.g., Martiny et al., 2006), applying both to terrestrial microbes and aquatic microbes mostly at large spatial scales of continents or regions. Drivers underlying spatial patterns are investigated, and generally correspond to changes in abiotic conditions (i.e., habitat characteristics). In the present topic, Mukhtar et al. demonstrate strong environmental filtration of microorganisms associated with plants suggesting a patchiness being strongly dependent on selection processes. Integrating space into account, Steven et al. demonstrated patchy distribution of bacterial communities in the soil due to local habitat distribution but also as a result of past disturbance. This article nicely demonstrates the scale of response of these communities both in space and time, and proposes that soil edaphic factors, climatic disturbances, and physical trampling are drivers of these biogeographical patterns. This article also provides an interesting example of integrating drivers occurring in the past for taking into account time-lags in species responses observed. One key question related to understanding species patterns concerns the possible local adaptation to patchy environments. Wang et al. reported here geographical variation of mineral elements of one cyanobacteria developing in the soil. This article proposes to study both the geographical variation of one microorganism species, and the related biochemical composition and mineral element contents. This original work helps to explore how the geographical variation of mineral content promotes the ecological adaptation of soil cyanobacteria. In most existing literature, biogeography has been applied at a large spatial scale, Schiro et al. present here a demonstration that biogeography can apply a very small spatial scale (scale of a field). They demonstrated the small-scale heterogeneity in fungi occurring in plant phyllosphere (and especially pathogens). This heterogeneity was described by local taxon-level selection to variable canopy environmental conditions, especially linked to climatic conditions, but also to a lesser extent by geographical position.

\section{LANDSCAPE ECOLOGY}

Landscape ecology provides a range of theories for understanding how landscape structure affects species coexistence. Methods specific to landscape ecology have been also developed to precisely characterize landscape metrics, analyze how landscape shapes species distribution and dispersal. Applications to microorganisms have mostly focused on one species of interest at a time, primarily pathogens (leading to the field of "landscape epidemiology"). Landscape epidemiology has been developed in all ecosystems on plant, animal or human pathogens, and even within the human body for predicting the development of infectious agents [see reviews of Holdenrieder et al. (2004) and Suzan et al. (2012)]. Use of landscape ecology concepts and methods in microbiology is at an early stage (see review of Mony et al.). In this topic, Mennicken et al. proposed an interesting study analyzing a recent theory of landscape ecology, the habitat amount hypothesis (Fahrig, 2013), on a large set of microorganism guilds and demonstrated for some of them the importance of forest cover in the landscape. Additionally, this article brings original evidence of time-lagged dispersal processes, by demonstrating that patch age affects the response of microorganisms to habitat amount. Integrating time into landscape studies offers new promising prospects in microbial ecology.

\section{METACOMMUNITY ECOLOGY}

Understanding the mechanisms of community assembly at landscape spatial scales is a key issue. The metacommunity framework assumes that community assembly results from four key processes: dispersal, species sorting, patch dynamics, and drift (Leibold et al., 2004). Metacommunity theory is a useful framework for predicting species coexistence, as illustrated in two articles in the topic. Zha et al. analyze how environmental selection, dispersal, and drift explain variation in composition of gut microbiota in fishes. In another study, Sokol et al. analyzed the metacommunity functioning of diatoms in Antarctica, and similarly demonstrated the effect of dispersal and species sorting in community assembly. Because many microorganisms have very short generation times, they are possibly experiencing more rapid evolutionary processes than macroorganisms. Integrating evolutionary processes in metacommunity models, O'Connor et al. analyze evolutionary rescue within a metacommunity framework and demonstrate that the history of antibiotic selection and dispersal modes influence diversification in microbial metacommunities. Metacommunity and landscape ecology are generally poorly linked (Almeida-Gomes et al., 2020), even in macroorganisms. Miller and Bohannan propose an adaptation of the metacommunity model that takes into account the permeability of the matrix to dispersal movement. Using this adapted model, they analyzed how species traits in microorganisms might promote species persistence in the matrix, with possible adaptation to human and animal-associated microbiomes. This study is a pioneering attempt to go beyond the patch-matrix binary vision of landscape and to integrate landscape heterogeneity into the metacommunity framework. 


\section{PERSPECTIVES}

Integrating landscape-scale drivers in community assembly for microorganisms is an important step toward a better understanding of species distribution, although the application of landscape ecology is still in its infancy. The application of landscape ecology to microorganisms has a number of potential applications, including in environmental restoration (such as that described by Gonçalves Selari et al. in this special feature).

The Mony et al. review provides examples of how theories and methods from landscape ecology can be applied successfully to microbes; importantly, it also highlights the knowledge gaps that interfere with such application, including our ignorance of many ecological processes operating at the landscape scale, such as microbial dispersal. It also stresses the need for adaptation of this framework to take into account microorganism characteristics and better integration of their real dispersion rates and realized niches. Landscape-microbe relationships are more complex, and less well-understood, than for macroorganisms involving a large range of response scales-from the classical landscape scale to micro landscapes-, nested spatial and temporal scales, and a unique case of biotic landscapes for hostassociated microorganisms. Microbial community composition and distribution are also often driven directly by their host distribution, and feedback loops between micro- and macroorganisms likely occur. If the landscape ecology framework in its broad sense (i.e., including biogeography and metacommunity concepts) provides a new avenue for testing ecological hypotheses

\section{REFERENCES}

Almeida-Gomes, M., Valente-Neto, F., Pacheco, E. O., Ganci, C. C., Leibold, M. A., Melo, A. S., et al. (2020). How does the landscape affect metacommunity structure? A quantitative review for lentic environments. Curr. Landsc. Ecol. Rep. 5, 68-75. doi: 10.1007/s40823-020-00049-6

Baas Becking, L. G. M. (1934). Geobiologie of Inleiding Tot de Milieukunde. The Hague: W. P. Van Stockum and Zoon.

Bahram, M., Peay, K. G., and Tedersoo, L. (2015). Local-scale biogeography and spatiotemporal variability in communities of mycorrhizal fungi. New Phytol. 205, 1454-1463. doi: 10.1111/nph.13206

Donaldson, G. P., Lee, S., and Mazmanian, S. K. (2016). Gut biogeography of the bacterial microbiota. Nat. Rev. Microbiol. 134, 20-32. doi: 10.1038/nrmicro3552

Fahrig, L. (2013). Rethinking patch size and isolation effects: the habitat amount hypothesis. Ann. J. Biogeogr. 40, 1649-1663. doi: 10.1111/jbi.12130

Hanski, I. (1994). A practical model of metapopulation dynamics. J. Anim. Ecol. 63, 151-162. doi: 10.2307/5591

Hanson, C. A., Fuhrman, J. A., Horner-Devine, C., and Martiny, J. B. H. (2012). Beyond biogeographic patterns: processes shaping the microbial landscape. Nat. Rev. Microbiol. 10, 497-506. doi: 10.1038/nrmicro2795

Holdenrieder, O., Pautasso, M., Weisberg, P. J., and Lonsdale, D. (2004). Tree diseases and landscape processes: the challenge of landscape pathology. Trends Ecol. Evol. 19, 446-452. doi: 10.1016/j.tree.2004. 06.003

Kraft, N. J. B., and Ackerly, D. D. (2014). “Assembly of plant communities," in Ecology and the Environment, ed R. K. Monson (New York, NY: SpringerVerlag), 67-88. doi: 10.1007/978-1-4614-7501-9_1

Leibold, M. A., Holyoak, M., Mouquet, N., Amarasekare, P., Chase, J. M., Hoopes, M. F., et al. (2004). The metacommunity concept: a framework for multiscale community ecology. Ecol. Lett. 7, 601-613. doi: 10.1111/j.1461-0248.2004. 00608.x on microbial community assembly, along with eco-evolutionary processes shaping communities, microbes provide also a unique and original case study that might considerably enrich the existing theoretical backgrounds and methods. Within a more holistic framework of understanding the application of landscape ecology to the microbial world is susceptible to modify our understanding not solely about the rules of microbial community assembly, but also for instance (i) eco-evolutionary processes and dynamics structuring microbial communities, (ii) macro-ecological processes resulting from changes of microbial communities, (iii) links between host and symbionts, all resulting from/to a better interpretation to which extend the neutral model of assembly is applicable rather than non-random influences of niche differentiation to the considered microbial community. The landscape of opportunities for future research in this field is wide.

\section{AUTHOR CONTRIBUTIONS}

CM wrote the first draft. All authors reviewed the text and gave final approval.

\section{FUNDING}

This research was funded by U.S. NSF award 2025118 (to $\mathrm{ML}$ ), CNRS EC2CO (to PV), and l'agence Française pour la Biodiversité project AGRIM (to CM).
MacArthur, R. H., and Wilson, E. O. (1967). The Theory of Island Biogeography. Princeton, NJ: Princeton University Press.

Martiny, J. B. H., Bohannan, B. J. M., Brown, J. H., Colwell, R. K., Fuhrman, J. A., Green, J. L., et al. (2006). Microbial biogeography: putting microorganisms on the map. Nat. Rev. Microbiol. 4, 102-112. doi: 10.1038/nrmicro1341

Suzan, G., Esponda, F., Carrasco-Hernandez, R., and Alonso Aguirre, A. (2012). "Habitat fragmentation and infectious disease ecology," in New Directions in Conservation Medicine: Applied Cases of Ecological Health, eds A. Alonso Aguirre, R. Ostfeld, and P. Daszak (New York, NY. Owford University Press).

Turner, M. G., Gardner, R. H., and O’Neill, R. V. (2001). Landscape Ecology in Theory and Practice. New York, NY: Springer-Verlag.

Wiens, J. A., Stenseth, N. C., Van Horne, B., and Anker Ims, R. (1993). Ecological mechanisms and landscape ecology. Oikos 66, 369-380. doi: 10.2307/3544931

Conflict of Interest: The authors declare that the research was conducted in the absence of any commercial or financial relationships that could be construed as a potential conflict of interest.

Publisher's Note: All claims expressed in this article are solely those of the authors and do not necessarily represent those of their affiliated organizations, or those of the publisher, the editors and the reviewers. Any product that may be evaluated in this article, or claim that may be made by its manufacturer, is not guaranteed or endorsed by the publisher.

Copyright (C) 2021 Mony, Bohannan, Leibold, Peay and Vandenkoornhuyse. This is an open-access article distributed under the terms of the Creative Commons Attribution License (CC BY). The use, distribution or reproduction in other forums is permitted, provided the original author(s) and the copyright owner(s) are credited and that the original publication in this journal is cited, in accordance with accepted academic practice. No use, distribution or reproduction is permitted which does not comply with these terms. 